\title{
INVARIANT SUBSPACES OF NONSELFADJOINT TRANSFORMATIONS
}

\author{
BY LOUIS DE BRANGES
}

Communicated by P. R. Halmos, April 17, 1963

This note comments on recent Russian results in Hilbert space. Macaev [9] has given a fundamental estimate of completely continuous transformations which have no nonzero spectrum. The same estimate is true of transformations with imaginary spectrum.

THEOREM I. Let $T$ be a densely defined transformation in a Hilbert space He such that $T^{*}$ has the same domain as $T$ and $T-T^{*}$ has a completely continuous extension. Suppose that

$$
T-T^{*} \subset 2 i \sum \operatorname{sgn} k c_{k} \bar{c}_{k},
$$

where $\left(c_{k}\right)$ is an orthogonal set in $\mathfrak{H}$, indexed by the odd integers, $\left\|c_{k+2}\right\| \leqq\left\|c_{k}\right\|$ for $k>0,\left\|c_{k-2}\right\| \leqq\left\|c_{k}\right\|$ for $k<0$, and

$$
\delta=\sum\left\|c_{k}\right\|^{2} /|k|<\infty .
$$

If the spectrum of $T$ is imaginary, then the spectrum of $\frac{1}{2}\left(T+T^{*}\right)$ is contained in the interval $[-2 \delta / \pi, 2 \delta / \pi]$.

If $a$ and $b$ are elements of a Hilbert space, $\bar{a} b$ is the inner product, $\bar{a} b=\langle b, a\rangle$, and $a \bar{b}$ is the linear transformation defined by $(a \bar{b}) c$ $=a(b c)$ for every $c$ in $\mathfrak{F C}$. The proof of Theorem $\mathrm{I}$ is similar to Macaev's except that it depends on the following new estimate of eigenvalues.

THEOREM II. Let $S$ be an everywhere defined and bounded transformation in a Hilbert space $\mathfrak{H}$, which has imaginary spectrum, such that

$$
S-S^{*}=2 i \sum b_{n} b_{n},
$$

where $\left(b_{n}\right)$ is an orthogonal set in $\mathfrak{F C}$ and $\sum\left\|b_{n}\right\|^{2}$ is finite. Then,

$$
S+S^{*}=2 \sum \operatorname{sgn} k a_{k} \bar{a}_{k},
$$

where $\left(a_{k}\right)$ is an orthogonal set in $\mathfrak{H}$, indexed by the odd integers, $\left\|a_{k+2}\right\| \leqq\left\|a_{k}\right\|$ for $k>0,\left\|a_{k-2}\right\| \leqq\left\|a_{k}\right\|$ for $k<0$, and

for every $k$.

$$
\left\|a_{k}\right\|^{2} \leqq(2 / \pi)\left(\sum\left\|b_{n}\right\|^{2}\right) /|k|
$$

Macaev [9] has given a fundamental existence theorem for invariant subspaces. It can be deduced directly from Theorem I without using, as he indicates, an additional estimate of resolvents. Neither 
boundedness nor a real spectrum is necessary in the statement of the theorem.

THEOREM III. Let $T$ be a densely defined transformation in a Hilbert space $\mathfrak{H C}$ such that $T^{*}$ has the same domain as $T$ and $T-T^{*}$ has a completely continuous extension of the form (1) where (2) holds. If $h$ is a given real number, there exists a closed subspace $\mathfrak{N}$ of $\mathfrak{H}$, which is invariant under the resolvents of $T$, such that the restriction of $T$ to $\mathfrak{T C}$ has its spectrum in the half-plane $x \leqq h$ and the restriction of $T^{*}$ to the orthogonal complement of TI has its spectrum in the half-plane $x \geqq h$.

Macaev's existence theorem is stated for transformations which are, in a technical sense, nearly selfadjoint. A similar existence theorem holds for transformations which are nearly unitary.

THEOREM IV. Let $T$ be an everywhere defined and bounded transformation in a Hilbert space $\mathfrak{H}$ which has an everywhere defined and bounded inverse. Suppose that

$$
T^{*} T-1=\sum \epsilon_{k} c_{k} \bar{c}_{k},
$$

where $\left(c_{k}\right)$ is an orthogonal set in $\mathcal{H}, \epsilon_{k}= \pm 1$ for every $k,\left\|c_{k+1}\right\| \leqq\left\|c_{k}\right\|$, and

$$
\sum \| c_{k}||^{2} /|k|<\infty \text {. }
$$

If $\alpha$ is a given real number, $0<\alpha<\pi$, then there exists a closed subspace IT of $\mathcal{H C}$ which is invariant under $T$ and $T^{-1}$, such that the restriction of $T$ to Tl has its spectrum in the sector $-\alpha \leqq \theta \leqq \alpha$, and the restriction of $T^{*}$ to the orthogonal complement of $\mathfrak{T C}$ has its spectrum in the complementary sector $\alpha \leqq \theta \leqq 2 \pi-\alpha$.

Invariant subspaces of this nature need not exist if the hypotheses of Theorem IV are not satisfied.

ThEOREM V. Let $\left(c_{k}\right)$ be an orthogonal set in a Hilbert space Te such that $\left\|c_{k+1}\right\| \leqq\left\|c_{k}\right\|<1$ for every $k, \lim c_{k}=0$, and (4) is not satisfied. Let $\epsilon_{k}= \pm 1$ for every $k$. Then there exists an everywhere defined and bounded transformation $T$ in $\mathfrak{H C}$, with an everywhere defined and bounded inverse, which satisfies (3), and the spectrum of the restriction of $T$ to every nonzero closed subspace invariant under $T$ and $T^{-1}$ is the full unit circle $|z|=1$.

The proof of Theorem $\mathrm{V}$ depends on the theory of translation invariance. If $W(x)$ is a complex valued function of integral $x$, consider the corresponding Hilbert space of functions $f(x)$ of integral $x$, such that

$$
\|f\|^{2}=\sum|f(n) / W(n)|^{2}<\infty .
$$


If $W(x) / W(x-1)$ and $W(x) / W(x+1)$ are bounded, the translation operator $T: f(x) \rightarrow f(x-1)$ is bounded and has a bounded inverse. Complete continuity of $T^{*} T-1$ means that

$$
\lim |W(x) / W(x-1)|=1
$$

as $|x| \rightarrow \infty$, and in this case the spectrum of the transformation is the unit circle. If $|W(x)|$ is increasing for negative $x$ and is decreasing for positive $x$, condition (4) is equivalent to

$$
\sum\left(1+n^{2}\right)^{-1} \log |W(n)|>-\infty .
$$

The proof of Theorem $\mathrm{V}$ is completed using a theorem of Levinson, as it is stated in [1].

In the situation of Theorem III, $T$ has an integral representation of the form

$$
T=\int h(t) d P(t)+\int P(t)\left(T-T^{*}\right) d P(t),
$$

where $P(x)$ is a nondecreasing function whose values are projections into invariant subspaces for the resolvents of $T$. The first term on the right is a selfadjoint transformation. The second term is an everywhere defined and bounded transformation with imaginary spectrum. The theory of this second integral is that of Gohberg and Kreln [6], except that it is not restricted to transformations which have the origin as the only point in the spectrum. The integration theory involves three distinct topics: (a) the uniqueness of transformations with given invariant subspaces, (b) the existence of sufficiently many invariant subspaces to characterize a given transformation, and (c) the existence of transformations with given invariant subspaces. Theorem I is the essential estimate in each case.

A fundamental problem is to determine the uniqueness of such integral representations. The essential difficulty is due to the lack of information about invariant subspaces of transformations whose spectrum is a point. In special cases the invariant subspaces are totally ordered by inclusion. Results of this nature are obtainable from the theory of Hilbert spaces of entire functions [2]. This theory contains implicitly a determination of the invariant subspaces of transformations $T$, with no nonzero spectrum, when $T-T^{*}$ has two dimensional range and its eigenvalues are not on the same side of the real axis. See [3] for the relation between Hilbert spaces of entire functions and invariant subspaces of transformations. In particular the results of [2] may be used to verify a conjecture of Kreyn, stated by Brodskir [5], that the real invariant subspaces of the above trans- 
formations are totally ordered by inclusion.

Unfortunately Theorems VI and VIII of [3] are erroneous as stated. Theorem VIII is easily corrected, but we can find no valid form of Theorem VI which does not leave a gap between the problem of invariant subspaces and factorization problems for operator valued analytic functions. What is false in Theorem VI is that $M(a, b, z)$, $M(b, c, z)$, and $M(a, c, z)$ need satisfy condition (4) there, which implies that the corresponding spaces have a trivial structure. As a result the existence of invariant subspaces is not known in all cases in which the $M(z)$ function can be factored.

Added in proof. The following hypothesis should be added to Theorem V. The orthogonal set $\left(c_{k}\right)$ is complete in $\mathcal{H} C$ unless there are only a finite number of positive $\epsilon_{k}$ or of negative $\epsilon_{k}$, in which case the orthogonal complement of the $c_{k}$ is of countably infinite dimension.

\section{REFERENCES}

1. L. de Branges, The a-local operator problem, Canad. J. Math. 11 (1959), 583-592.

2. - Some Hilbert spaces of entire functions. IV, Trans. Amer. Math. Soc. 105 (1962), 43-83.

3. - Some Hilbert spaces of analytic functions. I, Trans. Amer. Math. Soc., 106 (1963), 445-468.

4. - Perturbations of self-adjoint transformations, Amer. J. Math., 84 (1962), 543-560.

5. M. S. Brodski1, On the unicellularity of real Volterra operators, Dokl. Akad. Nauk SSSR 147 (1962), 1010-1012. (Russian)

6. I. C. Gohberg and M. G. KreYn, Completely continuous operators whose spectrum is concentrated at zero, Dokl. Akad. Nauk SSSR 128 (1959), 227-230. (Russian)

7. - On the theory of the triangular representation of non-selfadjoint operators, Dokl. Akad. Nauk SSSR 137 (1961), 1034-1037. (Russian)

8. - Volterra operators whose imaginary component belongs to a given class, Dokl. Akad. Nauk SSSR 139 (1961), 779-782. (Russian)

9. V. I. Macaev, On the class of completely continuous operators, Dokl. Akad. Nauk SSSR 139 (1961), 548-551. (Russian)

10. - Volterrra operators produced by perturbation of selfadjoint operators, Dokl. Akad. Nauk SSSR 139 (1961), 810-813. (Russian)

Purdue University 